\title{
Generation of the magnetic field in jets
}

\author{
V. Urpin ${ }^{1,2}$ \\ 1 Departament de Física Aplicada, Universitat d'Alacant, Ap. Correus 99, 03080 Alacant, Spain \\ e-mail: vadim.urpin@uv.es \\ 2 A.F. Ioffe Institute of Physics and Technology and Isaak Newton Institute of Chili, Branch in St. Petersburg, 194021 St. Petersburg, \\ Russia
}

Received 16 January 2006 / Accepted 17 April 2006

ABSTRACT

\begin{abstract}
We consider dynamo action under the combined influence of turbulence and large-scale shear in sheared jets. Shear can stretch turbulent magnetic field lines in such a way that even turbulent motions showing mirror symmetry become suitable for generation of a large-scale magnetic field. We derive the integral induction equation governing the behaviour of the mean field in jets. The main result is that sheared jets may generate a large-scale magnetic field if shear is sufficiently strong. The generated mean field is mainly concentrated in a magnetic sheath surrounding the central region of a jet, and it exhibits sign reversals in the direction of the jet axis. Typically, the magnetic field in a sheath is dominated by the component along the jet that can reach equipartition with the kinetic energy of particles. The field in the central region of jets has a more disordered structure.
\end{abstract}

Key words. magnetohydrodynamics (MHD) - ISM: jets and outflows - galaxies: jets - ISM: magnetic fields - gravitational lensing - magnetic fields

\section{Introduction}

It is widely believed that magnetic fields play an important role in the formation and propagation of astrophysical jets providing an efficient mechanism of collimation through magnetic tension forces (see, e.g., Hughes 1991; Blandford 1993; Königl \& Pudritz 1999). Polarization observations provide information on the orientation and degree of order of the magnetic field in jets. It appears that many jets can develop relatively highly organized magnetic structures (see, e.g., Cawthorne et al. 1993; Leppänen et al. 1995; Gabuzda 1999). For example, radio emission from the jets of NGC 4258 indicates that the magnetic field is oriented mainly along the jet axis but a noticeable toroidal component can also be presented (Krause \& Löhr 2004). The conventional estimate of the magnetic field strength in jets comes from the minimum energy argument and corresponds to approximate equipartition between magnetic and jet particle energy (see, e.g., Laing 1993). Recent observations of jets in TeV BL Lac objects confirm this estimate (Ghisellini et al. 2005). However, Kataoka \& Stawarz (2005) argue that the powerful jets in quasars and FR II objects can be far from the minimum energy condition, and the field strength very likely exceeds the equipartition value. Upper limits to the inverse Compton radiation of the jet in M 87 imposed by HESS and HEGRA Cerenkov Telescopes also indicate that the magnetic field cannot be weaker than the equipartition value (Stawarz et al. 2005).

To explain the observational data, various simplified models of three-dimensional magnetic structures have been proposed (see, e.g., Chan \& Henriksen 1980; Laing 1981, 1999). For example, Canvin et al. (2005) computed emission and polarization for a few different models of the jets in NGC 315 and concluded that both fully and partially ordered fields can produce the high fractional polarization observed. It seems (Laing 1999) that good agreement with observations can be obtained if the jet is considered as a cylindrical core with more or less uniform density and pressure surrounded by a shear layer. Possibly, the core and shear layer are both turbulent (see, e.g., Gabuzda 1999). The magnetic field has a substantial longitudinal component in the shear layer, but the field can be random or a transverse component dominates in the core region. Recent VLBI observations (Hirabayashi et al. 1998; Gabuzda et al. 2004) indicate that the transverse field may have a substantial toroidal component in some objects.

The mechanisms responsible for generation of the magnetic field in jets are still unclear. Since the origin of jets is probably relevant to MHD-processes in magnetized plasma, their magnetic fields could be generated during the process of jet formation (see, e.g., Blandford \& Payne 1982; Romanova \& Lovelace 1992; Koide et al. 1998). However, as mentioned, jets are possibly turbulent, and turbulent dissipation should lead to a rapid decay of any initially ordered fields if the mean-field dynamo does not operate. Besides, the toroidal component becomes dominant as the flow expands (Begelman et al. 1984) and, under certain conditions, this component can be unstable thereby decollimating the jet (see, e.g., Spruit et al. 1997; Begelman 1998). Therefore, some mechanisms for generating the magnetic field should certainly be operative in jets. One of these possibilities has been considered by Honda \& Honda (2002), who argued that the stream can generate a toroidal field that participates in self-pinching the plasma in a fully relativistic jet consisting of electrons, positrons, and a small portion of ions.

The magnetic field in jets can also be generated by some dynamo mechanism. It is plausible that jets are turbulent making the turbulent dynamo a suitable candidate. This point is argued by Stawarz et al. (2005) for the particular case of the jet in M 87 where the field strength is probably stronger than the equipartition value. The origin of turbulence can be attributed to different instabilities arising in jets. The classical Kelvin-Helmholtz instability seems to be the dominating factor of destabilization in the most simplified jet model with one bulk velocity and a 
narrow interface with the external medium. This instability has been studied by many authors both in linear (e.g., Blandford \& Pringle 1976; Ray 1981; Payne \& Cohn 1985; Zhao et al. 1992) and nonlinear regimes (Bodo et al. 1994; Koide et al. 1996; Nishikava et al. 1997; Hardee et al. 1998). In more refined models, however, other hydrodynamic instabilities can manifest themselves leading to production of turbulence in jets (Urpin 2002a; Alloy et al. 2002). Gvaramadze et al. (1984) proposed the model where the field is generated due to the combined influence of helical turbulence and regular flow that stretches a seed magnetic field. In this model, however, the field grows superexponentially only during the initial stage but decays eventually, sothat a true dynamo is replaced by a temporal growth of the field.

In this paper, we consider the turbulent dynamo action that can be responsible for generation of the magnetic field in jets. Our model is based on the shear-driven dynamo action associated with turbulent shear flows (see, e.g., Urpin 1999, 2002b). Shear stretches turbulent magnetic field lines in the direction of a mean flow, which in turn generates the additional component of the mean electromotive force, which is proportional to the production of shear and magnetic field. Plausibly, the flow inside jets is sheared and turbulent, and the shear-driven dynamo can be in action there. There is observational evidence that the jet structure is rather complex and that different bulk velocities can be represented inside the jet; for more details see the discussion in Hanasz \& Sol (1996) and Stawarz \& Ostrowski (2002). Theoretical models of the jet formation also predict the existence of a nonvanishing transverse gradient in the jet velocity (see, e.g., Melia \& Königl 1989; Königl \& Kartje 1994; Sol et al. 1989).

In the present paper, we show that the shear-driven dynamo can naturally explain the observed magnetic structure of jets. In Sect. 2, we derive the main equations governing the magnetic field in sheared turbulent jets. The geometry and growth rate of the generated field is then considered in Sect. 3. A brief summary of our results is finally presented in Sect. 4.

\section{The mean-field electrodynamics of jets}

We model the jet as a cylindrical flow in the z-direction with radius $R$. Plasma inside the jet has a velocity $\boldsymbol{V}=V(r) \boldsymbol{e}_{z}$, and $r$, $\varphi, z$ are the cylindrical coordinates with $\boldsymbol{e}_{r}, \boldsymbol{e}_{\varphi}$, and $\boldsymbol{e}_{z}$ the corresponding unit vectors. For the sake of simplicity, we assume that the jet velocity is subrelativistic. Without loss of generality, we can assume that density $\rho$ is constant inside the jet. The Reynolds number is large in jets and, possibly, the plasma of sheared jets is turbulent. Variations in the flux, which are observed in many jets, are sometimes interpreted in terms of this turbulence (e.g., Marscher et al. 1992; Massaro et al. 1999).

We can represent the magnetic field $\mathcal{B}$ and the velocity $\boldsymbol{u}$ as a sum of the mean and fluctuating parts, $\mathcal{B}=\boldsymbol{B}+\boldsymbol{b}$ and $\boldsymbol{u}=\boldsymbol{V}+\boldsymbol{v}$, where $\boldsymbol{B}$ and $\boldsymbol{V}$ are the mean field and velocity, respectively. We neglect dissipative effects in the induction equation and assume the field to be frozen in plasma. Then, averaging the induction equation, we have for the mean field

$$
\frac{\partial \boldsymbol{B}}{\partial t}=\nabla \times(\boldsymbol{V} \times \boldsymbol{B})+\nabla \times \mathcal{E},
$$

where

$\mathcal{E}=\langle\boldsymbol{v} \times \boldsymbol{b}\rangle$

is the mean electromotive force; $\langle\ldots\rangle$ denote ensemble averaging. In what follows, however, we will need the sign of dissipative terms to choose the integration path properly when calculating Fourier integrals. Generally, a weak dissipation in jets can be provided by either electrical resistivity (if plasma is collisional) or some plasma mechanisms, such as Landau damping (if plasma is collisionless).

We consider the mean electromotive force $\mathcal{E}$ using a quasilinear approximation. In this approximation, mean quantities are governed by equations including non-linear effects in fluctuating terms, whilst the linearized equation is used for the fluctuating quantities (Krause \& Rädler 1980). A quasilinear approximation is accurate enough, for example, to describe an ensemble of waves with relatively high frequencies and small amplitudes. Then, the linearized induction equation for the fluctuating magnetic field reads

$\frac{\partial \boldsymbol{b}}{\partial t}=\nabla \times(\boldsymbol{V} \times \boldsymbol{b})+\nabla \times(\boldsymbol{v} \times \boldsymbol{B})$.

If $\boldsymbol{V}=V(r) \boldsymbol{e}_{z}$, then we have

$\frac{\partial \boldsymbol{b}}{\partial t}+V(r) \frac{\partial \boldsymbol{b}}{\partial z}-\boldsymbol{e}_{z} b_{r} V^{\prime}(r)=\boldsymbol{A}$

where

$\boldsymbol{A}=(\boldsymbol{B} \cdot \nabla) \boldsymbol{v}-(\boldsymbol{v} \cdot \nabla) \boldsymbol{B}-\boldsymbol{B} \nabla \cdot \boldsymbol{v}$,

and $V^{\prime}=\mathrm{d} V / \mathrm{d} r$.

Equation (4) can be solved by making use of a partial Fourier transformation. Since coefficients in Eq. (4) do not depend on $\varphi$ and $z$ we make initial transformations in these coordinates. The fluctuating magnetic field can be represented as

$\boldsymbol{b}(\boldsymbol{r}, t)=\sum_{m} \int \mathrm{d} k_{z} \hat{\boldsymbol{b}}_{1}\left(r, m, k_{z}, t\right) \mathrm{e}^{-\mathrm{i} m \varphi-\mathrm{i} k_{z} z}$,

where $m$ is an integer. Then,

$\hat{\boldsymbol{b}}_{1}\left(r, m, k_{z}, t\right)=\frac{1}{(2 \pi)^{2}} \int \mathrm{d} \varphi \mathrm{d} z \mathrm{e}^{\mathrm{i} m \varphi+\mathrm{i} k_{z} z} \boldsymbol{b}(\boldsymbol{r}, t)$,

and the equation for $\hat{\boldsymbol{b}}_{1}$ reads

$\frac{\partial \hat{\boldsymbol{b}}_{1}}{\partial t}-\mathrm{i} k_{z} V \hat{\boldsymbol{b}}_{1}-\boldsymbol{e}_{z} V^{\prime} \hat{b}_{1 r}=\hat{\boldsymbol{A}}_{1}$

where $\hat{A}_{1}\left(r, m, k_{z}, t\right)$ is the corresponding Fourier amplitude of $\boldsymbol{A}(\boldsymbol{r}, t)$. Substituting $\hat{\boldsymbol{b}}_{1}=\mathrm{e}^{\mathrm{i} k_{z} V t} \hat{\boldsymbol{b}}_{2}$, we obtain the equation for $\hat{\boldsymbol{b}}_{2}$ that does not contain the advective term,

$\frac{\partial \hat{\boldsymbol{b}}_{2}}{\partial t}-\boldsymbol{e}_{z} V^{\prime} \hat{b}_{2 r}=\mathrm{e}^{-\mathrm{i} k_{z} V t} \hat{\boldsymbol{A}}_{1}$.

This equation can be solved by Fourier transformation in $t$. Introducing

$$
\begin{aligned}
\hat{\boldsymbol{b}}\left(r, m, k_{z}, \omega\right) & =\frac{1}{2 \pi} \int \mathrm{d} t \mathrm{e}^{-\mathrm{i} \omega t} \hat{\boldsymbol{b}}_{2}\left(r, m, k_{z}, t\right) \\
& =\frac{1}{2 \pi} \int \mathrm{d} t \mathrm{e}^{-\mathrm{i}\left(\omega+k_{z} V\right) t} \hat{\boldsymbol{b}}_{1}\left(r, m, k_{z}, t\right),
\end{aligned}
$$

we obtain the following expression from Eq. (9)

$\hat{\boldsymbol{b}}=-\frac{\mathrm{i}}{\omega} \hat{\boldsymbol{A}}\left(r, m, k_{z}, \omega\right)-\frac{V^{\prime}}{\omega^{2}} \hat{A}_{r}\left(r, m, k_{z}, \omega\right) \boldsymbol{e}_{z}$.

Expression (10) is not a complete Fourier transform of $\boldsymbol{b}(\boldsymbol{r}, t)$ since it depends on the radial coordinate. Note that, in reality, 
Eq. (10) does not contain singularities because neglected dissipative terms would result in small negative imaginary corrections to $\omega$, so we would have $\omega-\mathrm{i} 0$ instead of $\omega$ in singular terms. Here, $\pm \mathrm{i} 0$ denotes a positive (or negative) contribution to $\omega$ caused by weak dissipative effects. The sign of this small imaginary term is important when calculating Fourier integrals.

The solution for a fluctuating magnetic field reads

$\boldsymbol{b}(\boldsymbol{r}, t)=\sum_{m} \int \frac{\mathrm{d} \omega \mathrm{d} k_{z}}{\mathrm{i} \omega} \mathrm{e}^{\mathrm{i}\left(\omega+k_{z} V\right) t-\mathrm{i} m \varphi-\mathrm{i} k_{z} z}\left(\hat{\boldsymbol{A}}-\frac{\mathrm{i} V^{\prime}}{\omega} \hat{A}_{r} \boldsymbol{e}_{z}\right)$.

Substituting this expression into the definition of $\mathcal{E}$, we obtain

$$
\begin{aligned}
\mathcal{E}= & \sum_{m} \int \frac{\mathrm{d} \omega \mathrm{d} k_{z}}{\mathrm{i}(2 \pi)^{3} \omega} \mathrm{d} \varphi_{1} \mathrm{~d} z_{1} \mathrm{~d} t^{\prime} \mathrm{e}^{\mathrm{i} \omega\left(t-t^{\prime}\right)-\mathrm{i} k_{z}\left[z-z_{1}-V\left(t-t^{\prime}\right)\right]} \\
& \times \mathrm{e}^{-\mathrm{i} m\left(\varphi-\varphi_{1}\right)}\left\langle\boldsymbol{v}(\boldsymbol{r}, t) \times\left[\boldsymbol{A}\left(\boldsymbol{r}_{1}, t^{\prime}\right)-\frac{\mathrm{i} V^{\prime}}{\omega} A_{r}\left(\boldsymbol{r}_{1}, t^{\prime}\right) \boldsymbol{e}_{z}\right]\right\rangle,
\end{aligned}
$$

where $\boldsymbol{r}_{1}=\left(r, \varphi_{1}, z_{1}\right)$. Taking into account that summation over $m$ and integration over $\mathrm{d} k_{z}$ yield the corresponding $\delta$-functions, we can simplify expression (12):

$$
\begin{array}{r}
\mathcal{E}=\int \frac{\mathrm{d} \omega \mathrm{d} t^{\prime}}{2 \pi \mathrm{i} \omega} \mathrm{e}^{\mathrm{i} \omega\left(t-t^{\prime}\right)}\left\langle\boldsymbol{v}(\boldsymbol{r}, t) \times\left[\boldsymbol{A}\left(\boldsymbol{r}^{\prime}, t^{\prime}\right)\right.\right. \\
\left.\left.-\frac{\mathrm{i} V^{\prime}}{\omega} A_{r}\left(\boldsymbol{r}^{\prime}, t^{\prime}\right) \boldsymbol{e}_{z}\right]\right\rangle\left.\right|_{\boldsymbol{r}^{\prime}=\boldsymbol{r}-\boldsymbol{V}\left(t-t^{\prime}\right)} .
\end{array}
$$

Integrals over $\mathrm{d} \omega$ can now be calculated using the known integrals if we note that $\omega$ has a small imaginary part caused by dissipation. We have (see Gradshtein \& Ryzhik 1965)

$\frac{1}{2 \pi} \int_{-\infty}^{\infty} \frac{\mathrm{e}^{-\mathrm{i} p x} \mathrm{~d} x}{(\mathrm{i} x+\beta)^{v}}=\frac{(-p)^{\nu-1}}{\Gamma(v)} e^{\beta p} \quad$ if $p<0$,

and 0 if $p>0$. The parameter $\beta$ in this integral is small in our model because it is caused by dissipation. Then, the expression for $\mathcal{E}$ transforms into

$$
\begin{aligned}
\mathcal{E}= & \int_{-\infty}^{t} \mathrm{~d} t^{\prime}\left\langle\boldsymbol{v}(\boldsymbol{r}, t) \times\left[\boldsymbol{A}\left(\boldsymbol{r}^{\prime}, t^{\prime}\right)\right.\right. \\
& \left.\left.+\left(t-t^{\prime}\right) V^{\prime} A_{r}\left(\boldsymbol{r}^{\prime}, t^{\prime}\right) \boldsymbol{e}_{z}\right]\right\rangle\left.\right|_{\boldsymbol{r}^{\prime}=\boldsymbol{r}-\boldsymbol{V}\left(t-t^{\prime}\right)} .
\end{aligned}
$$

Since $\boldsymbol{A}\left(\boldsymbol{r}^{\prime}, t^{\prime}\right)$ depends on the turbulent velocity (see Eq. (5)) we can now calculate $\mathcal{E}$ if specifying the correlation properties of turbulence. For the sake of simplicity, we assume turbulence to be locally isotropic and homogeneous with the correlation tensor given by

$$
\left\langle\hat{v}_{i}\left(\omega^{\prime}, \boldsymbol{k}^{\prime}\right) \hat{v}_{j}\left(\omega^{\prime \prime}, \boldsymbol{k}^{\prime \prime}\right)\right\rangle=P k_{i}^{\prime} k_{j}^{\prime} \delta\left(\boldsymbol{k}^{\prime}+\boldsymbol{k}^{\prime \prime}\right) \delta\left(\omega^{\prime}+\omega^{\prime \prime}\right),
$$

where $P=P\left(\omega^{\prime}, \boldsymbol{k}^{\prime}\right)$ is the spectral function and $(i, j)$ denote Cartesian components. This correlation tensor corresponds to acoustic turbulence (see, e.g., Rüdiger 1989), which seems to be plausible in a supersonic jet flow. For instance, sound waves can be generated by the Kelvin-Helmholtz instability at the jet boundary and then propagate through the jet volume generating fluctuations of the velocity and density (Payne \& Cohn 1985).

Since correlation tensor (16) is of particular simplicity in Cartesian components, it is convenient to represent the turbulent velocities $\boldsymbol{v}(\boldsymbol{r}, t)$ and $\boldsymbol{v}\left(\boldsymbol{r}^{\prime}, t^{\prime}\right)$ in Eq. (15) in terms of Fourier integrals with Cartesian wavevectors as

$\boldsymbol{v}(\boldsymbol{r}, t)=\int \mathrm{d} \omega^{\prime} \mathrm{d} \boldsymbol{k}^{\prime} \mathrm{e}^{\mathrm{i} \omega^{\prime} t-\mathrm{i} \boldsymbol{k}^{\prime}} \hat{\boldsymbol{v}}\left(\boldsymbol{k}^{\prime}, \omega^{\prime}\right)$.
Substituting this expression into Eq. (15), we obtain, after ensemble averaging,

$$
\begin{array}{r}
\mathcal{E}=\int_{-\infty}^{t} \mathrm{~d} t^{\prime} \int \mathrm{d} \omega^{\prime} \mathrm{d} \boldsymbol{k}^{\prime} \mathrm{e}^{\mathrm{i} \omega^{\prime}\left(t-t^{\prime}\right)-\mathrm{i} \boldsymbol{k}^{\prime}\left(\boldsymbol{r}-\boldsymbol{r}^{\prime}\right)} P\left(\omega^{\prime}, \boldsymbol{k}^{\prime}\right) \\
\times\left[\boldsymbol{E}_{1}-\left(t-t^{\prime}\right) V^{\prime} \boldsymbol{e}_{z} \times \boldsymbol{E}_{2}\right],
\end{array}
$$

where

$\boldsymbol{E}_{1}=-\boldsymbol{k}^{\prime} \times\left[\mathrm{i} k^{\prime 2} \boldsymbol{B}+\left(\boldsymbol{k}^{\prime} \cdot \nabla^{\prime}\right) \boldsymbol{B}\right]$,

$\boldsymbol{E}_{2}=\boldsymbol{e}_{z} \times \boldsymbol{k}^{\prime}\left\{\mathrm{i} k_{r}^{\prime}\left(\boldsymbol{k}^{\prime} \cdot \boldsymbol{B}\right)-\mathrm{i} k^{\prime 2} B_{r}-\left[\left(\boldsymbol{k}^{\prime} \cdot \nabla^{\prime}\right) \boldsymbol{B}\right]_{r}\right\}$,

and $\nabla^{\prime}=\left(\partial / \partial r^{\prime}, \partial / r \partial \varphi^{\prime}, \partial / \partial z^{\prime}\right)$. Note that $\boldsymbol{B}$ in these expressions is a function of $\boldsymbol{r}^{\prime}$ and $t^{\prime}$.

Since turbulence is locally isotropic and homogeneous in a co-moving frame, the spectral function $P\left(\omega^{\prime}, \boldsymbol{k}^{\prime}\right)$ should be an even function of the frequency $\omega^{\prime \prime}=\omega^{\prime}-k_{z}^{\prime} V$ measured in a comoving frame, i.e. we have $P\left(\omega^{\prime}-k_{z}^{\prime} V, \boldsymbol{k}^{\prime}\right)=P\left(-\omega^{\prime}+k_{z}^{\prime} V, \boldsymbol{k}^{\prime}\right)$. Then, denoting $P\left(\omega^{\prime}-k_{z}^{\prime} V, \boldsymbol{k}^{\prime}\right)=G\left(\omega^{\prime \prime}, \boldsymbol{k}^{\prime}\right)$, we can transform Eq. (17) into

$$
\begin{aligned}
\mathcal{E}= & \int \mathrm{d} \omega^{\prime \prime} \mathrm{d} \boldsymbol{k}^{\prime} G\left(\omega^{\prime \prime}, \boldsymbol{k}^{\prime}\right) \int_{-\infty}^{t} \mathrm{~d} t^{\prime} \mathrm{e}^{\mathrm{i} \omega^{\prime \prime}\left(t-t^{\prime}\right)} \\
& \times\left.\left[\boldsymbol{E}_{1}\left(\boldsymbol{r}^{\prime}, t^{\prime}\right)-\left(t-t^{\prime}\right) V^{\prime} \boldsymbol{E}_{2}\left(\boldsymbol{r}^{\prime}, t^{\prime}\right)\right]\right|_{\boldsymbol{r}^{\prime}=\boldsymbol{r}-\boldsymbol{V}\left(t-t^{\prime}\right)} .
\end{aligned}
$$

Averaging of $\boldsymbol{E}_{1}$ and $\boldsymbol{E}_{2}$ over directions of $\boldsymbol{k}^{\prime}$ yields

$\boldsymbol{E}_{1}=-\frac{k^{\prime 2}}{3} \nabla^{\prime} \times \boldsymbol{B}, \boldsymbol{E}_{2}=-\frac{k^{\prime 2}}{3} \boldsymbol{e}_{z} \times\left(\nabla^{\prime} \boldsymbol{B}\right)_{r}$,

where $\left(\nabla^{\prime} \boldsymbol{B}\right)_{r}=\nabla^{\prime} B_{r}-\boldsymbol{e}_{\varphi} B_{\varphi} / r^{\prime}$. Finally, the expression for the mean electromotive force reads

$$
\begin{aligned}
\mathcal{E}= & -\int_{-\infty}^{t} \mathrm{~d} t^{\prime} F\left(t-t^{\prime}\right)\left[\nabla^{\prime} \times \boldsymbol{B}\right. \\
& \left.-\left(t-t^{\prime}\right) V^{\prime} \boldsymbol{e}_{z} \times\left(\nabla^{\prime} \boldsymbol{B}\right)_{r}\right]\left.\right|_{r^{\prime}=\boldsymbol{r}-\boldsymbol{V}\left(t-t^{\prime}\right)},
\end{aligned}
$$

where

$$
F(t)=\frac{4 \pi}{3} \int \mathrm{d} \omega \mathrm{d} k k^{4} G(\omega, \boldsymbol{k}) \mathrm{e}^{\mathrm{i} \omega t} .
$$

Note that the mean electromotive force given by Eq. (20) is nonlocal in our approach since the turbulent magnetic field is determined by its previous evolution under the influence of shear.

\section{Generation of a large-scale field in jets}

We adopt the simplest model of a jet assuming that shear is relatively weak in the central region but is stronger in a shear layer near the jet surface, $r=R$. If $\Delta r$ is the thickness of a shear layer, then the shear-driven dynamo operates in the region $R \geq r \geq R-\Delta r$. Simulations indicate that often the thickness of a region with strong shear can be much smaller than the jet radius (see Alloy et al. 1999a,b). At that point, we will not specify the radial dependence of $V(r)$ because this dependence seems to be rather uncertain from both theoretical and observational points of view. However, we show that the proposed mechanism can generate the magnetic field for any dependence $V(r)$.

In a stationary jet, the solution of the mean induction Eq. (1) can be represented as

$\boldsymbol{B}(\boldsymbol{r}, t)=\boldsymbol{B}(r) \mathrm{e}^{\gamma t-\mathrm{i} K_{z} z-\mathrm{i} M \varphi}$,

where $K_{z}$ is a wavevector of the magnetic field in the $z$-direction, $M$ the azimuthal wavenumber, and $\gamma$ the growth rate. Solution (22) describes spiral magnetic waves. 
We are particularly interested in the generation of large-scale fields with not very large $M$. Since the thickness of a shear layer is typically smaller than $R$, we can neglect terms of the order of $1 / r$ compared to $\partial / \partial r$ in Eq. (1). Then, the $r$-component of Eq. (1) reads

$$
\begin{aligned}
\frac{\partial B_{r}}{\partial t}+V(r) \frac{\partial B_{r}}{\partial z}=\int_{0}^{\infty} \mathrm{d} \xi F(\xi)\left[\Delta^{\prime} B_{r}\left(\boldsymbol{r}^{\prime}, t-\xi\right)\right. \\
\left.\quad-\xi V^{\prime}(r) \frac{\partial^{2}}{\partial r^{\prime} \partial z^{\prime}} B_{r}\left(\boldsymbol{r}^{\prime}, t-\xi\right)\right]_{\boldsymbol{r}^{\prime}=\boldsymbol{r}-\boldsymbol{V} \xi}
\end{aligned}
$$

where $\Delta^{\prime}$ is the Laplacian with the primed coordinates. Substituting dependence (22) for $B_{r}(r)$, we obtain

$$
\begin{array}{r}
{\left[\gamma-\mathrm{i} K_{z} V(r)\right] B_{r}(r)=\int_{0}^{\infty} \mathrm{d} \xi F(\xi) \mathrm{e}^{-\left[\gamma-\mathrm{i} K_{z} V(r)\right] \xi}} \\
\times\left[\frac{\mathrm{d}^{2} B_{r}}{\mathrm{~d} r^{2}}-K_{\perp}^{2} B_{r}+i \xi V^{\prime}(r) K_{z} \frac{\mathrm{d} B_{r}}{\mathrm{~d} r}\right],
\end{array}
$$

where $K_{\perp}^{2}=K_{z}^{2}+M^{2} / r^{2}$. Note that the radial dependence of the azimuthal component of $\boldsymbol{B}$ satisfies the same equation in our model. Integrating Eq. (24) over $\mathrm{d} \xi$, we have

$\frac{\mathrm{d}^{2} B_{r}}{\mathrm{~d} r^{2}}-\mathrm{i} K_{z} V^{\prime}(r) \frac{\lambda_{\mathrm{T}}}{\mu_{\mathrm{T}}} \frac{\mathrm{d} B_{r}}{\mathrm{~d} r}-\left(K_{\perp}^{2}+\frac{\Gamma}{\mu_{\mathrm{T}}}\right) B_{r}=0$

where

$\mu_{\mathrm{T}}=\frac{4 \pi}{3} \Gamma \int \frac{G(\omega, \boldsymbol{k})}{\omega^{2}+\Gamma^{2}} k^{4} \mathrm{~d} \omega \mathrm{d} k$

$\lambda_{\mathrm{T}}=\frac{4 \pi}{3} \int \frac{\left(\omega^{2}-\Gamma^{2}\right) G(\omega, \boldsymbol{k})}{\left(\omega^{2}+\Gamma^{2}\right)^{2}} k^{4} \mathrm{~d} \omega \mathrm{d} k$,

and $\Gamma=\Gamma(r)=\gamma-\mathrm{i} K_{z} V(r)$. The coefficient $\mu_{\mathrm{T}}$ represents a nonlocal magnetic viscosity in a turbulent shear flow, and the coefficient $\lambda_{\mathrm{T}}$ describes a qualitatively new turbulent kinetic process that can be responsible for the generation of the mean field. Note that, in our nonlocal model, turbulent kinetic coefficients depend on the rate of a mean process that is the principle difference to any local theory like a two-scale approximation. The kinetic coefficients are complex in our model since $\Gamma$ is complex. It is convenient to represent $B_{r}(r)$ as

$B_{r}(r)=f(r) \exp \left(\frac{\mathrm{i}}{2} K_{z} \int V^{\prime}\left(r^{\prime}\right) \frac{\lambda_{\mathrm{T}}}{\mu_{\mathrm{T}}} \mathrm{d} r^{\prime}\right)$.

Then, the equation for $f(r)$ reads

$$
\begin{aligned}
\frac{\mathrm{d}^{2} f}{\mathrm{~d} r^{2}}- & {\left[K_{\perp}^{2}+\frac{\Gamma}{\mu_{\mathrm{T}}}-\frac{1}{4} K_{z}^{2} V^{\prime 2}(r) \frac{\lambda_{\mathrm{T}}^{2}}{\mu_{\mathrm{T}}^{2}}\right.} \\
& \left.-\frac{i}{2} K_{z} \frac{\mathrm{d}}{\mathrm{d} r}\left(V^{\prime}(r) \frac{\lambda_{\mathrm{T}}}{\mu_{\mathrm{T}}}\right)\right] f=0 .
\end{aligned}
$$

The coefficients $\mu_{\mathrm{T}}$ and $\lambda_{\mathrm{T}}$ depend on $\Gamma$ and, hence, on $r$, so these dependences are determined by the spectral function. As mentioned, the origin of turbulent motions, as well as their spectrum, are rather uncertain in jets. Turbulent motions can be caused, for example, by acoustic waves generated due to the Kelvin-Helmholtz instability (see, e.g., Payne \& Cohn 1985). In this paper, we assume as a certainty that turbulence is acoustic and choose the simplest possible dependence of $G(\omega, \boldsymbol{k})$ on $\omega$,

$G(\omega, \boldsymbol{k})=\frac{G(\boldsymbol{k})}{\omega^{2}+\tau^{-2}}$, where $\tau$ is the characteristic correlation timescale of turbulence. Dependence (30) corresponds to a velocity correlation tensor exponentially decreasing with time,

$\left\langle v_{i}(\boldsymbol{r}, t) v_{j}(\boldsymbol{r}, t+\Delta t)\right\rangle \propto \mathrm{e}^{-\Delta t / \tau}$

(see, e.g., Rüdiger 1989). Then, the kinetic coefficients are

$\mu_{\mathrm{T}}=\frac{\tau v_{\mathrm{T}}^{2}}{1+\Gamma \tau}, \quad \lambda_{\mathrm{T}}=-\frac{\tau^{2} v_{\mathrm{T}}^{2}}{(1+\Gamma \tau)^{2}}$,

where $v_{\mathrm{T}}$ is the characteristic turbulent velocity,

$v_{\mathrm{T}}^{2}=\frac{8}{9} \pi^{2} \tau \int G(\boldsymbol{k}) k^{4} \mathrm{~d} k$

Substituting expressions (32) into Eq. (29) and taking into account that $\Gamma^{\prime}=-\mathrm{i} K_{z} V^{\prime}$, we obtain

$\frac{\mathrm{d}^{2} f}{\mathrm{~d} r^{2}}+q^{2}(r) f=0$

where

$q^{2}(r)=-\frac{\Gamma}{\mu_{\mathrm{T}}}-K_{\perp}^{2}+\frac{3}{4} \frac{K_{z}^{2} \tau^{2} V^{\prime 2}}{(1+\Gamma \tau)^{2}}-\frac{\mathrm{i}}{2} \frac{K_{z} \tau V^{\prime \prime}}{1+\Gamma \tau}$.

To solve Eq. (34) one needs the corresponding boundary conditions. For the sake of simplicity, we assume that plasma inside and outside of the shear layer is highly conductive and that the mean-field does not penetrate into the surrounding medium. Then, function $f(r)$ should be vanishing at $r=R$ and $r=R-\Delta r$. The main qualitative conclusions are the same for other possible boundary conditions.

To estimate the eigenvalues of Eq. (34), we can use an integral method similar to the one proposed by Chandrasekhar (1960). Since $f$ is complex, we multiply Eq. (34) by the complex conjugate function $f^{*}$ and integrate over the whole region where the magnetic field is generated. Then, we have

$\int_{R-\Delta r}^{R}\left|\frac{\mathrm{d} f}{\mathrm{~d} r}\right|^{2} \mathrm{~d} r-\int_{R-\Delta r}^{R} q^{2}(r)|f|^{2} \mathrm{~d} r=0$

for the chosen boundary conditions. Both $|\mathrm{d} f / \mathrm{d} r|^{2}$ and $|f|^{2}$ are positive quantities over the integration domain, but $q^{2}(r)$ is complex. Splitting Eq. (36) into the real and imaginary parts, we obtain

$$
\begin{array}{r}
\int_{R-\Delta r}^{R}\left|\frac{\mathrm{d} f}{\mathrm{~d} r}\right|^{2} \mathrm{~d} r=\int_{R-\Delta r}^{R} \operatorname{Re} q^{2}(r)|f|^{2} \mathrm{~d} r \\
\int_{R-\Delta r}^{R} \operatorname{Im} q^{2}(r)|f|^{2} \mathrm{~d} r=0 .
\end{array}
$$

By applying the mean value theorem, Eqs. (37) and (38) can be transformed into

$\int_{R-\Delta r}^{R} \operatorname{Re} q^{2}(r) \mathrm{d} r=\frac{\Delta r}{(\Delta R)^{2}}$
$\left|f\left(r_{2}\right)\right|^{2} \int_{R-\Delta r}^{R} \operatorname{Im} q^{2}(r) \mathrm{d} r=0$

where

$\frac{\Delta r}{(\Delta R)^{2}}=\left.\int_{R-\Delta r}^{R}\left|\frac{\mathrm{d} f}{\mathrm{~d} r}\right|^{2} \mathrm{~d} r|| f\left(r_{1}\right)\right|^{-2}$, 
$\Delta R \sim \Delta r$ is the characteristic radial lengthscale of the magnetic field, and $r_{1}$ and $r_{2}$ are some mean points within the shear layer. Equations (39) and (40) can be combined into

$\int_{R-\Delta r}^{R} q^{2}(r) \mathrm{d} r=\frac{\Delta r}{(\Delta R)^{2}}$

Substituting expression (35), we obtain the dispersion equation for dynamo modes

$\int_{R-\Delta h}^{R}\left[\Gamma \tau(1+\Gamma \tau)-\frac{3 K_{z}^{2} \ell^{2} \tau^{2} V^{\prime 2}}{4(1+\Gamma \tau)^{2}}+\frac{i K_{z} \ell^{2} \tau V^{\prime \prime}}{2(1+\Gamma \tau)}\right] \mathrm{d} r=-\ell^{2} Q^{2} \Delta r$,

where $V^{\prime \prime}=\mathrm{d}^{2} V / \mathrm{d} r^{2}$, and

$Q^{2}=\frac{1}{(\Delta R)^{2}}+\frac{1}{\Delta r} \int_{R-\Delta r}^{R} K_{\perp}^{2} \mathrm{~d} r \approx \frac{1}{(\Delta R)^{2}}+K_{z}^{2}+\frac{M^{2}}{R^{2}}$,

where $Q$ is the characteristic wavevector of dynamo waves, and $\ell=\tau v_{\mathrm{T}}$ is the lengthscale of turbulence.

To estimate the eigenvalues of Eq. (43), we initially consider the simplest model assuming that shear is approximately linear within the shear layer, $R \geq r \geq R-\Delta r$. In this case, the mean velocity can be represented as

$V(r)=V_{0}(R-r) / \Delta r$,

where $V_{0}$ is the velocity in the jet core, $r<R-\Delta r$. Then, Eq. (43) yields

$\mathrm{i} \int_{\tau \Gamma_{\mathrm{i}}}^{\tau \Gamma_{\mathrm{e}}}\left[x(1+x)-\frac{3 K_{z}^{2} \ell^{2} \tau^{2} V^{\prime 2}}{4(1+x)^{2}}\right] \mathrm{d} x=K_{z} V_{0} \tau \ell^{2} Q^{2}$,

where $\Gamma_{\mathrm{e}} \equiv \Gamma(R)=\gamma$ and $\Gamma_{\mathrm{i}} \equiv \Gamma(R-\Delta R)=\gamma-\mathrm{i} K_{z} V_{0}$ are the values of $\Gamma$ at the outer and inner boundaries of the shear layer. Integrating Eq. (46), we obtain

$$
\begin{aligned}
& (1+\gamma \tau)\left(\gamma-\mathrm{i} K_{z} V_{0}\right)+\frac{\mathrm{i}}{2} K_{z} V_{0}+\frac{\ell^{2}}{\tau} Q^{2} \\
& \quad-\frac{\tau}{3} K_{z}^{2} V_{0}^{2}\left[1+\frac{(3 \ell / 2 \Delta R)^{2}}{(1+\gamma \tau)\left(1+\gamma \tau-i \tau K_{z} V_{0}\right)}\right]=0
\end{aligned}
$$

The term proportional to $(3 \ell / 2 \Delta R)^{2}$ is small in the mean-field theory and can be neglected compared to 1 in Eq. (47). Then, the dispersion equation simplifies

$(1+\gamma \tau)\left(\gamma-\mathrm{i} K_{z} V_{0}\right)+\frac{\mathrm{i}}{2} K_{z} V_{0}-\frac{\tau}{3} K_{z}^{2} V_{0}^{2}+\frac{\ell^{2}}{\tau} Q^{2}=0$.

Splitting $\gamma$ into real and imaginary parts, $\gamma=\mathrm{i} \gamma_{\mathrm{I}}+\gamma_{\mathrm{R}}$, we obtain two equations for $\gamma_{\mathrm{I}}$ and $\gamma_{\mathrm{R}}$ from Eq. (48)

$\left(1+2 \gamma_{\mathrm{R}} \tau\right)\left(\gamma_{I}-\frac{1}{2} K_{z} V_{0}\right)=0$,

$\gamma_{\mathrm{R}}\left(1+\gamma_{\mathrm{R}} \tau\right)-\gamma_{\mathrm{I}} \tau\left(\gamma_{\mathrm{I}}-K_{z} V_{0}\right)-\frac{\tau}{3} K_{z}^{2} V_{0}^{2}+\frac{\ell^{2}}{\tau} Q^{2}=0$.

Equation (49) yields

$\gamma_{\mathrm{I}}=\frac{1}{2} K_{z} V_{0}$

Then, we have from Eq. (50)

$\left(\tau \gamma_{\mathrm{R}}\right)^{2}+\tau \gamma_{\mathrm{R}}-\left(\frac{\tau^{2}}{12} K_{z}^{2} V_{0}^{2}-\ell^{2} Q^{2}\right)=0$.
The roots of this equation are

$\tau \gamma_{\mathrm{R} 1,2}=-\frac{1}{2} \pm \sqrt{\frac{1}{4}+\left(\frac{\tau^{2}}{12} K_{z}^{2} V_{0}^{2}-\ell^{2} Q^{2}\right)}$

One of the roots is positive, so the corresponding dynamo mode is growing if

$\frac{\tau^{2}}{12} K_{z}^{2} V_{0}^{2}>\ell^{2} Q^{2}$

Estimating $Q^{2} \approx 1 /(\Delta R)^{2}+K_{z}^{2}$ and assuming that the azimuthal wavelength is much larger than $\Delta R$, we can represent Eq. (54) as

$K_{z} \Delta R \frac{V_{0}}{v_{\mathrm{T}}}>2 \sqrt{3}\left(1-\frac{12 v_{\mathrm{T}}^{2}}{V_{0}^{2}}\right)^{-1 / 2}$.

This condition cannot be fulfilled if $V_{0}<2 \sqrt{3} v_{\mathrm{T}}$, and the dynamo does not operate in such jets. However, it is plausible that the mean velocity is much larger than the turbulent velocity in jets, $V_{0} \gg 2 \sqrt{3} v_{\mathrm{T}}$, and condition (55) can be satisfied for a wide range of dynamo modes with

$\lambda_{z}<\frac{\pi V_{0}}{\sqrt{3} v_{\mathrm{T}}} \Delta R$

where $\lambda_{z}=2 \pi / K_{z}$ is the wavelength in the $z$-direction. Therefore, the maximum longitudinal wavelength of the dynamo-generated magnetic structure in jets is of the order of $\Delta R\left(V_{0} / v_{\mathrm{T}}\right)$.

The generation time, $t_{*}=1 / \gamma_{\mathrm{R}}$, is given by

$t_{*}=2 \tau\left[-1+\sqrt{1+\frac{K_{z}^{2} \tau^{2}}{3}\left(V_{0}^{2}-12 v_{\mathrm{T}}^{2}\right)-\frac{4 \ell^{2}}{(\Delta R)^{2}}}\right]^{-1}$.

For dynamo waves with a relatively short wavelength, $\lambda_{z}<$ $2 \pi \ell\left(V_{0} / v_{\mathrm{T}}\right)\left(\right.$ or $\left.V_{0} \gg 1 / \tau K_{z}\right)$, the growth time is

$t_{*} \sim \frac{2 \sqrt{3}}{K_{z} V_{0}}$.

In the limit of a large wavelength, $\lambda_{z}>2 \pi \ell\left(V_{0} / v_{\mathrm{T}}\right.$ ) (but still satisfying condition (55)), Eq. (57) yields

$t_{*} \sim \frac{12 \tau}{K_{z}^{2} \ell^{2}} \frac{v_{\mathrm{T}}^{2}}{V_{0}^{2}}$.

The dynamo modes with the maximum possible wavelength $\left(\sim \Delta R\left(V_{0} / v_{\mathrm{T}}\right)\right)$ grow on the slowest timescale $t_{*} \sim \tau(\Delta R / \ell)^{2}$.

It is seen from this consideration that generation of the meanfield is determined by a velocity difference between the boundaries of a shear layer rather than by the details of the velocity profile. Therefore, our results can be generalized for any velocity profile in a relatively simple way. We can split Eq. (43) into the real and imaginary parts and then obtain equations analogous to Eqs. (51) and (52) by applying the mean value theorem. The only difference to Eqs. (51) and (52) is that the equations for a more complicated velocity profile will contain the value of a flow velocity on some mean point $V\left(r_{*}\right)\left(R>r_{*}>R-\Delta r\right)$ instead of $V_{0}$. Correspondingly, the growth rate and the generation condition will be given by Eqs. (53) and (54), respectively, with the replacement $V_{0} \rightarrow V\left(r_{*}\right)$. Therefore, a particular shape of the velocity profile appears to be unimportant in our model, and large-scale magnetic fields can be generated in any jets with a strong shear. 


\section{Discussion}

We have considered the turbulent dynamo action in jets. The main result is that even the simplest turbulent motions showing the mirror symmetry become suitable for the generation of a large-scale magnetic field in the presence of shear. An amplification of the mean field takes place due to non-local terms that appear in the mean electromotive force and are caused by shear stresses. The considered mechanism of generation is qualitatively different from the conventional turbulent alpha-dynamo that, apart from the lack of the mirror symmetry of turbulence, also requires large-scale stratification. Due to its simplicity, the proposed mechanism is well adopted to the physical conditions in jets because the presence of both shear and turbulence seems to be plausible in a jet flow.

Unfortunately, neither available observational data nor theoretical modelling provide reliable information concerning the velocity profile in jets. However, the generation of a large-scale magnetic field can take place for any velocity profile, which is an attractive feature of our model. The only necessary condition of the considered dynamo is the presence of a sufficiently strong shear satisfying condition (54). This condition can be fulfilled in many jets or, at least, in a fraction of their volume. This dynamo mechanism generates the field in jets on a very short timescale that can be comparable to the turnover time of turbulence $\tau$ and that is typically much shorter than the lifetime of jets. Therefore, the generation can most likely reach a saturation level when the dynamo works in the nonlinear regime.

We considered generation of the radial component of $\boldsymbol{B}$ since this component is the most important one in the shear-driven dynamo. Generally, two other components can be stronger but their evolution is determined entirely by the behaviour of $B_{r}$. We can estimate $B_{z}$ from the $z$-component of the mean induction equation, equating the dissipative and stretching terms. Then,

$B_{z} \sim \frac{V^{\prime}(\Delta R)^{2}}{\mu_{\mathrm{T}}} B_{r} \sim K_{z} \Delta R\left(\frac{V}{v_{\mathrm{T}}}\right)^{2} B_{r}$,

and the longitudinal magnetic field is always stronger than the radial one. The azimuthal magnetic field can be estimated from the divergence condition and can vary within a wide range depending on the longitudinal wavelength and $M$. For not very large $M \neq 0, B_{\varphi}$ is typically stronger than the radial field. Our linear analysis does not allow proper estimation of the saturation magnetic field but, most likely, the strongest field component can reach equipartition with the kinetic energy of jet particles.

In our model, a dynamo generates a large-scale field in the layer of the thickness $\Delta r$ near the jet surface. The shear-driven dynamo is much less efficient in the core region, $r<R-\Delta r$. However, if the jet is turbulent, the small-scale turbulent dynamo can amplify small-scale magnetic fields in the core region even if the shear is negligible. The magnetic Reynolds number is large in jets, and turbulent motions caused, for example, by instabilities stretch and distort the field lines increasing thereby the energy of generated small-scale magnetic fields rapidly (see, e.g., Schekochihin et al. 2001). This random field reaches equipartition with the energy of turbulent motions on a very short timescale $\sim \tau$. Since turbulent motions are less energetic than the mean flow, we can expect that random fields in the central region are typically weaker than the large-scale magnetic field in a magnetic sheath surrounding the jet. The characteristic lengthscale of turbulent magnetic fields in the central region can be shorter than $\ell$.

\section{References}

Alloy, M. A., Ibánez, J. M., Marti, J. M., \& Müller, E. 1999a, ApJS, 122, 151 Alloy, M. A., Ibánez, J. M., Marti, J. M., \& Müller, E. 1999b, ApJ, 523, L125 Alloy, M. A., Ibánez, J. M., Miralles, J. A., \& Urpin, V. 2002, A\&A, 396, 693 Begelman, M. 1998, ApJ, 493, 291

Begelman, M., Blandford, R., \& Rees, M. 1984, Rev. Mod. Phys., 56, 255

Blandford, R. 1993, in Astrophysical Jets, ed. D. Burgarella, M. Livio, \& C. P. O’Dea (Cambridge: Cambridge University Press)

Blandford, R., \& Payne, D. 1982, MNRAS, 199, 883

Blandford, R., \& Pringle, J. E. 1976, MNRAS, 176, 443

Bodo, G., Massaglia, S., Ferrari, A., \& Trussoni, E. 1994, A\&A, 283, 655

Canvin, J. R., Laing, R. A., Bridle, A. H., \& Cotton, W. D. 2005, MNRAS, 363 , 1223

Cawthorne, T., Wardle, J., Roberts, D., Gabuzda, D., \& Brown, L. 1993, ApJ, 416, 496

Chan, K. L., \& Henriksen, R. N. 1980, ApJ, 241, 534

Chandrasekhar, S. 1960. Proc. Nat. Acad. Sci., 46, 253

Gabuzda, D. 1999, in Plasma Turbulence and Energetic Particles in Astrophysics, ed. M. Ostrowski, \& R. Schlickeiser, Krakow: Obserwatorium Astronomiczne, Universytet Jagiellonski

Gabuzda, D., Murray, E., \& Cronin, P. 2004, MNRAS, 351, 89L

Ghisellini, G., Tavecchio, F., \& Chiaberge, M. 2005, A\&A, 432, 401

Gradshtein, I., \& Ryzhik, I. 1965, Table of integrals, series, and productions (New York: Academic Press)

Gvaramadze, V., Lominadze, J., Ruzmaikin, A., Sokoloff, D., \& Shukurov, A. 1984, Ap\&SS, 140, 165

Hanasz, M., \& Sol, H. 1996, A\&A, 315, 355

Hardee, P., Rosen, A., Hughes, P., \& Comer Duncan, G. 1998, ApJ, 500, 599

Hirabayashi, H., Hirosawa, H., Kobayashi, H., et al. 1998, Science, 281, 1825

Honda, M., \& Honda, Y. 2002, ApJ, 569, L39

Hughes, P. A. 1991, Beams and Jets in Astrophysics (Cambridge: Cambridge University Press)

Kataoka, J., \& Stawarz, L. 2005, ApJ, 622, 797

Koide, S., Nishikawa, K.-I., \& Mutel, R.-I. 1996, ApJ, 463, L71

Koide, S., Shibata, K., \& Kudoh, T. 1998, ApJ, 495, L63

Königl, A., \& Kartje, A. 1994, ApJ, 434, 446

Königl, A., \& Pudritz, R. 1999, in Protostars and Planets III, ed. V. Mannings, A. Boss, \& S. Russell (Tucson: University of Arizona Press)

Krause, F., \& Rädler, K.-H. 1980, Mean-field magnetohydrodynamics and dynamo theory (Berlin: Akademie-Verlag)

Krause, M., \& Löhr, A. 2004, A\&A, 420, 115

Laing, R. A. 1981, ApJ, 248, 87

Laing, R. A. 1993, in Astrophysical Jets, ed. D. Burgarella, M. Livio, \& C. P. O'Dea (Cambridge: Cambridge University Press)

Laing, R. A. 1999, in Energy Transport in Radio Galaxies and Quasars, ed. P. Hardee, A. Bridle, \& J. Zensus (San Francisco: ASP)

Leppänen, K., Zensus, A., \& Diamond, P. 1995, AJ, 110, 2479

Marscher, A., Gear, W., \& Travis, J. 1992, in Variability of Blazars, ed. E. Valtaoja, \& M. Valtonen (Cambridge: Cambridge University Press)

Massaro, E., Cipriani, P., Nesci, R., \& Tosti, G. 1999, in Plasma Turbulence and Energetic Particles in Astrophysics, ed. M. Ostrowski, \& R. Schlickeiser, Krakow: Obserwatorium Astronomiczne, Universytet Jagiellonski

Melia, F., \& Königl, A. 1989, ApJ, 340, 162

Nishikawa, K.-I., Koide, S., Sakai, J., et al. 1998, ApJ, 498, 166

Payne, D., \& Cohn, H. 1985, ApJ, 291, 635

Ray, T. 1981, MNRAS, 196, 195

Romanova, M., \& Lovelace, R. 1992, A\&A, 262, 26

Rüdiger, G. 1989, Differential rotation and stellar convection (New York: Gordon \& Breach)

Schekochihin, A., Cowley, S., Maron, J., \& Malyshkin, L. 2001, Phys. Rev. E, 65,6305

Sol, H., Pelletier, G., \& Asseo, E. 1989, MNRAS, 237, 411

Spruit, H., Foglizzo, T., \& Stehle, R. 1997, MNRAS, 288, 333

Stawarz, L., \& Ostrowski, M. 2002, ApJ, 578, 763

Stawarz, L., Siemiginowska, A., Ostrowski, M., \& Sikora, M. 2005. ApJ, 626, 120

Urpin, V. 1999, A\&A, 347, L47

Urpin, V. 2002a, A\&A, 385, 14

Urpin, V. 2002b, Phys. Rev. E, 65, 6301

Zhao, J.-H., Burus, J., Hardee, P., \& Norman, M. 1992. ApJ, 387, 69 